\title{
Stacked Polymeric Multimode Waveguide Arrays for Two-Dimensional Optical Interconnects
}

\author{
Joon-Sung Kim and Jang-Joo Kim
}

\begin{abstract}
Two-dimensional (2-D) polymeric multimode waveguide arrays with two reflection-mirrors have been fabricated for optical interconnects between 2-D arrayed vertical-cavity surfaceemitting lasers and detectors. Contact printing lithography was adopted for simple and low-cost process using ultraviolet-curable epoxy-based polymers. Fabricated waveguides were diced of the same size and stacked one by one with lateral positional errors less than $\pm \mathbf{2 0} \mu \mathrm{m}$. Two kinds of mirrors were fabricated: single-reflection mirror and double-reflection mirror. Double-reflected mirrors resulted in lower losses with $1.2 \mathrm{~dB}$ than single reflected mirrors with $2.1 \mathrm{~dB}$. The average insertion losses of 16-channel arrayed waveguides with two single-reflection mirrors and with two double-reflection-mirrors were measured to be 6.1 and $4.4 \mathrm{~dB}$ for 6-cm-long waveguides at a wavelength of $830 \mathrm{~nm}$, respectively. The crosstalk between the waveguides was less than $-25 \mathrm{~dB}$. The characteristics of the waveguide arrays are good enough for applications to optical interconnects.
\end{abstract}

Index Terms-Contact printing lithography, double-reflection mirror, stacked polymeric waveguide arrays, two-dimensional (2-D) optical interconnects.

\section{INTRODUCTION}

I $\mathrm{N}$ recent years, much attention has been paid to research on the board-level parallel optical interconnects because of their high-bandwidth, high density, and low crosstalk within the system. Two-dimensional (2-D) parallel optical interconnects using 2-D arrays of vertical-cavity surface-emitting lasers (VCSELs) and photodiodes (PDs) have large connectivity between elements in computing and switching systems.

Two-dimensional optical interconnects within a short distance for chip-to-chip interconnects have been developed using various methods such as free-space microoptic components [1], [2] and plastic optical fibers (POFs) [3], [4]. Free-space optical interconnection is ideal for a high number of densely spaced channels. A 256-channel free-space optical interconnect using microoptic components has been demonstrated using 256 VCSELs and PDs, respectively [1]. However, it has a bulky and complicated structure because it consists of lenses and prisms or mirrors. Moreover, it is susceptible to vibration. Interconnects using POFs have been proposed over the last few years [3], [4]. POFs have flexibility and a low transmission loss for low-cost short-distance optical interconnects so that

Manuscript received July 31, 2003; revised November 17, 2003. This work was supported by the Ministry of Commerce, Industry and Energy and by the BK 21 program.

J.-S. Kim is with the Department of Materials Science and Engineering, Kwangju Institute of Science and Technology, 500-712 Gwangju, Korea.

J.-J. Kim is with the Organic Photonics Lab, School of Materials Science and Engineering, Seoul National University, 151-744 Seoul, Korea (e-mail: jjkim@snu.ac.kr).

Digital Object Identifier 10.1109/JLT.2004.824523

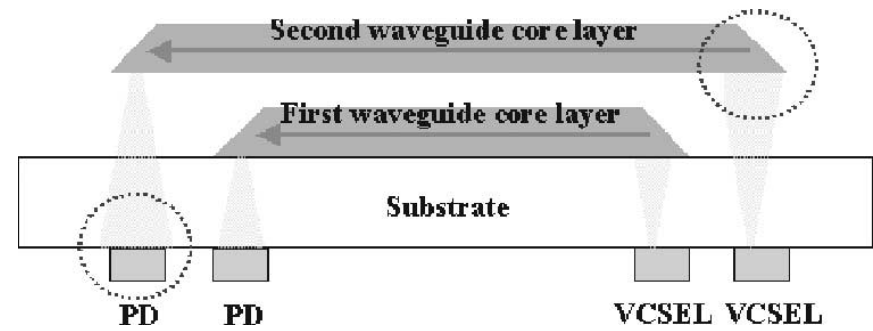

Fig. 1. Illustration of the structural problem of the conventional 2-D stacked waveguides with mirrors. Distance between the VCSEL or PD and waveguides increases as the number of the stacked layers increases, resulting in the reduced coupling efficiency.

this method is useful to board-to-board interconnection. In chip-level application, POFs have been embedded in printed circuit boards [4]. POFs, however, are hard to handle in a range of very short interconnection length of several centimeters without bulkiness.

Polymeric optical waveguide is one of the candidates for interchip interconnects because direct chip-to-chip optical interconnects can eliminate optical fiber and connectors, which are attractive for low-cost assembly [5], [6]. In chip-level interconnects $45^{\circ}$ mirrors are required at the end of waveguide as $90^{\circ}$ out-of-plane optical deflectors for the coupling with VCSELs and PDs. Two-dimensional interconnects can be fabricated by stacking arrayed waveguides layer by layer. However, the stack of waveguides causes coupling losses because the coupling efficiencies between VCSELs and mirrors, and between mirrors and PDs, are dependent on the vertical distance between them, as shown in Fig. 1. This problem limits the number of stacked layers and leads to the large variation of optical losses. Because of the reason, 2-D interconnects using optical waveguides with mirrors have not been reported yet, even though the stacking optical waveguides without mirrors were reported [7].

In this paper, we report the fabrication of novel stacked polymeric multimode waveguide arrays, which have a core size of $50 \times 50 \mu \mathrm{m}^{2}$ with two reflecting mirrors for 2-D optical interconnects. The lateral and vertical spacing of waveguides are 250 and $600 \mu \mathrm{m}$, respectively. For this purpose, one-dimensional (1-D) planar waveguide arrays are fabricated first, and the diced 1-D arrays are stacked to form 2-D waveguide arrays for optical interconnects, as illustrated in Fig. 2. Integrated turning mirrors have many applications in integrated optical systems because the $90^{\circ}$ directional change of light is essential to compact photonic devices [8]. We adopted the double-reflection mirrors with air medium for high reflectivity [9]. One of the advantages of the arrayed waveguides with mirrors is relatively uniform insertion loss through the optical channels because the deviation of the insertion loss is not dependent on the location of mirrors. 


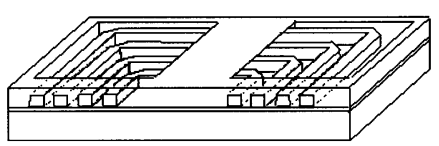

(a)

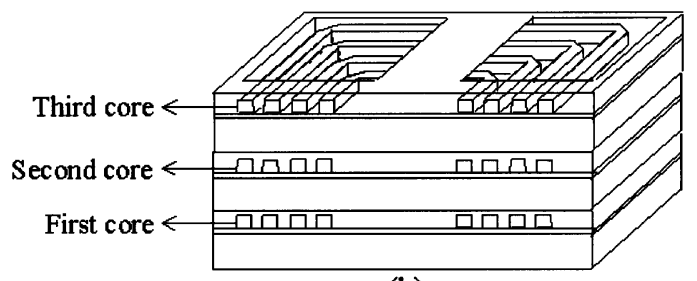

(b)

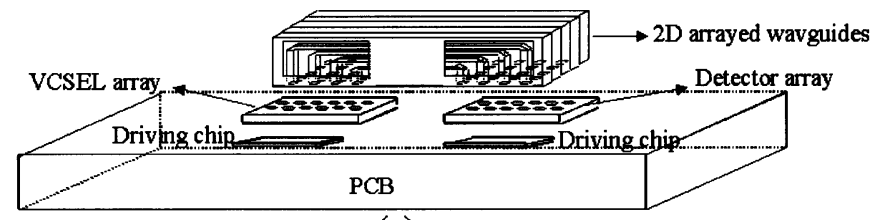

(c)

Fig. 2. Illustration of the fabrication process of 2-D stacked polymeric waveguide array for optical interconnects: (a) 1-D arrayed waveguides, (b) stacking process, and (c) the architecture of 2-D optical interconnects.

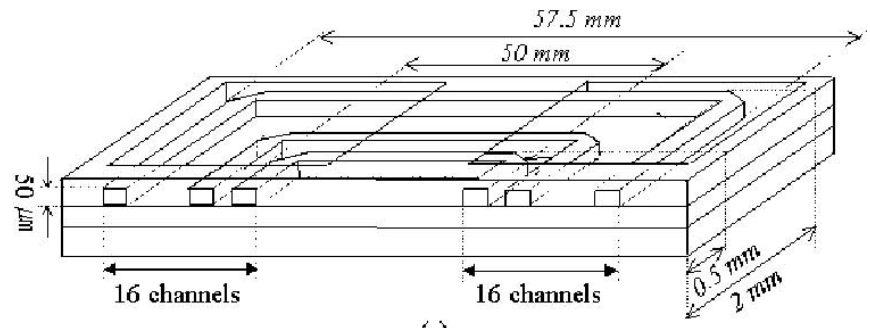

(a)

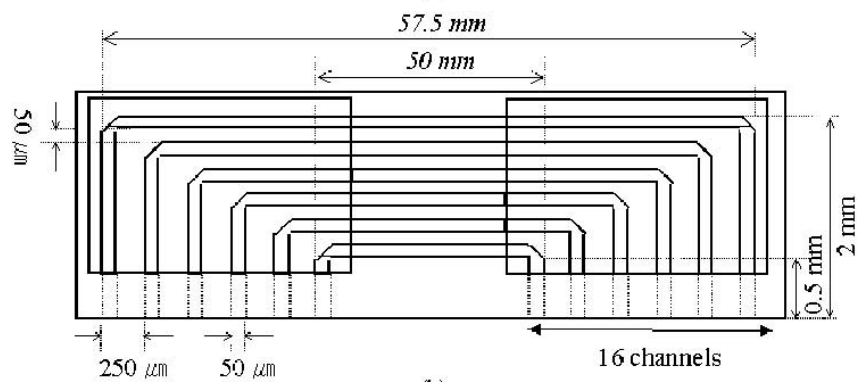

(b)

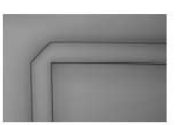

(c)

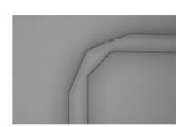

(d)
Fig. 3. (a) Schematic diagram and (b) top view of the arrayed waveguides and pictures of (c) the single-reflection mirror and (d) the double-reflection mirror.

\section{DESIGN}

Fig. 3 shows a schematic diagram for the arrayed waveguides and the reflecting mirrors. Each of the waveguide arrays has 16 channels with two reflecting mirrors. The waveguides in the arrays have the cross-sectional dimension of $50 \times 50 \mu \mathrm{m}^{2}$, and the lateral spacing between the waveguides is $250 \mu \mathrm{m}$ at the input and output. But the spacing between the waveguides located between mirrors is $50 \mu \mathrm{m}$.

The difference of the optical pass length between the longest and the shortest is less than $10.5 \mathrm{~mm}(61.5-51 \mathrm{~mm})$ in the

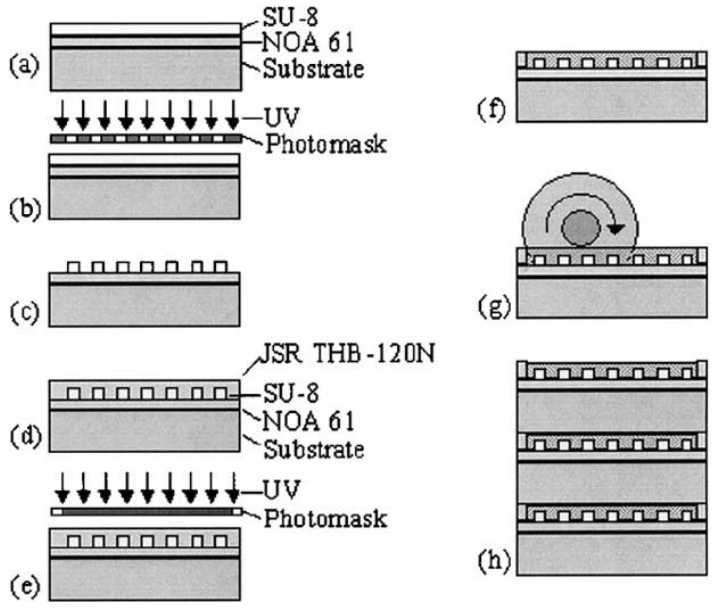

Fig. 4. Illustration of the fabrication processes for the arrayed waveguides using contact printing lithography. (a) Film formation of lower cladding and core layers. (b) Photolithography using a photomask. (c) Postbake and development. (d) Upper cladding layer coating. (e) Photolithography of upper cladding layer. (f) Development and hard bake. (g) Dicing process. (h) Stacking process of the arrayed waveguides.

array. We designed two types of micromirrors: single-reflection and double-reflection. The double-reflection mirror is expected to have higher reflectivity than the single-reflection mirror [9]. In order to improve the reflection efficiency of the mirrors, the upper cladding layer near the reflecting mirrors was etched out to form air cladding.

\section{FABRICATION}

The fabrication procedure of the arrayed polymeric waveguides for 2-D optical interconnects is illustrated in Fig. 4. The fabrication steps mainly consist of a conventional lithography to fabricate planar waveguide arrays and the stacking of the arrayed waveguides diced using a diamond blade. A $20-\mu$ m-thick NOA61 film was spin-coated on a 500- $\mu$ m-thick glass substrate as an undercladding layer. NOA61 is an ultraviolet (UV)-curable epoxy adhesives. The coated film was exposed to UV light and baked on a hot plate at $140{ }^{\circ} \mathrm{C}$ for $1 \mathrm{~h}$ in air.

A $50-\mu \mathrm{m}$-thick SU-8 film was spin-coated on the undercladding layer as the core layer and soft-baked at $65{ }^{\circ} \mathrm{C}$ for 5 min followed by $95{ }^{\circ} \mathrm{C}$ for $20 \mathrm{~min}$. We used the SU-8 polymer as the core layer because it has the low optical loss below $0.25 \mathrm{~dB} / \mathrm{cm}$ at the wavelength of $830 \mathrm{~nm}$, as well as the thermal and chemical stability [10]. The waveguide patterns were defined by the contact printing photolithography using UV light. Following the exposure, the film was baked at $65{ }^{\circ} \mathrm{C}$ for 2 min with a further baking at $95{ }^{\circ} \mathrm{C}$ for $5 \mathrm{~min}$ on a hot plate to selectively cross-link the exposed area of the film. The patterns were developed in propylene glycol methyl ether acetate (PGMEA) for $5 \mathrm{~min}$ followed by hard baking on a hot plate at $150{ }^{\circ} \mathrm{C}$ for $1 \mathrm{~h}$. JSR THB-120N (commercially available from the JSR company) was spin-coated on the top of the waveguides as the upper cladding layer and soft-baked at $110^{\circ} \mathrm{C}$ for $5 \mathrm{~min}$. The rectangular upper cladding patterns were defined by the contact printing photolithography using UV light to form air cladding at the mirrors. Photopatterning of the upper cladding layer is important to increase the refractive index difference between the core and the cladding layer to increase 


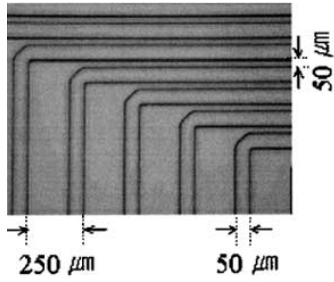

(a)

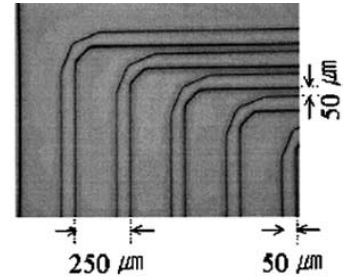

(b)

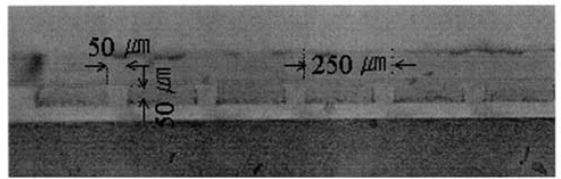

(c)

Fig. 5. Top views of arrayed waveguides with (a) the single-reflection mirrors and (b) the double-reflection mirrors. (c) The view of the cross section.

the reflection efficiency of the mirrors. Following the exposure, the upper cladding layer was developed in THB-D1(TMAHaq with surfactant) for $8 \mathrm{~min}$ and rinsed in distillated water followed by hard baking on a hot plate at $150{ }^{\circ} \mathrm{C}$ for $1 \mathrm{~h}$. The refractive indexes of the lower cladding, core, and upper cladding layer are $1.542,1.577$, and 1.525 at the wavelength of $830 \mathrm{~nm}$, respectively. The fabricated waveguide arrays in the wafer are diced of the same size by diamond saw and stacked using a fixture that has the same dimension as the waveguide arrays. A UV-curable acryl adhesive was coated and cured on the three sides of the stacked waveguide arrays to fix them.

\section{RESULTS}

Fig. 5 shows the top views and the cross-section of the fabricated waveguide array. The cross-section of the waveguide is approximately $50 \times 50 \mu \mathrm{m}^{2}$ with a nearly vertical sidewall. A four-layer polymer waveguide array for the optical interconnects using a two-dimensional VCSEL array is shown in Fig. 6.

The vertical and lateral positional error of the stacked waveguide arrays are about $\pm 5 \mu \mathrm{m}$ and $\pm 20 \mu \mathrm{m}$, respectively, due to passive alignment using the fixture. It can be reduced by a precision alignment using a camera and an actuator. The size variation of individual modules can be below $\pm 2 \mu \mathrm{m}$, which is defined by the positional error of the dicing machine [11]. The vertical spacing between the stacked arrays is $600 \mu \mathrm{m}$, which can be adjusted by controlling the thickness of the substrate or the cladding layer.

The coupling loss between VCSEL and waveguide due to the positional error can be estimated using the Lambercian model for $\mathrm{LD}$ as follows [12]:

$$
\eta=\frac{P_{w}}{P_{s}}=\left[1-\left(\cos \theta_{\max }\right)^{m+1}\right] \quad\left(\frac{A_{\mathrm{w}}}{A_{\mathrm{s}}}>1\right)
$$

where $P_{w}$ is the coupling power between VCSEL and waveguide and $P_{s}$ is the source power. $\cos \theta_{\max }$ is related to numerical aperture (NA) of a waveguide. $A_{\mathrm{w}}$ and $A_{\mathrm{s}}$ are the area of a waveguide and input mode size, which are $50 \mu \mathrm{m}^{2}$ and a circle with $20 \mu \mathrm{m}$ in diameter in our case, respectively. The value $m$ is related to the divergence angle of a VCSEL by

$$
\frac{P}{P_{s}}=\cos \theta^{m}
$$

where $P_{s}$ is the source power of VCSEL and $P$ is the half-power of $P_{s}$, which means that $P / P_{s}$ is 0.5 at divergence angle. The VCSEL used in this paper has a full-angle beam divergence of $12^{\circ}$ at the $-3 \mathrm{~dB}$ power points, which in turn gives the $m$ value of 126. The calculated coupling efficiency can be converted to the coupling loss $\left(L_{\mathrm{c}}\right)$ by

$$
L_{c}(\mathrm{~dB})=-10 \log (\eta) \quad(0 \leq \eta \leq 1) .
$$

The calculated coupling loss using the above equations is smaller than $2 \mathrm{~dB}$ with the positional error of $\pm 20 \mu \mathrm{m}$.

The arrayed waveguides were butt-coupled to an 830 -nm-wavelength laser light using a 50/125- $\mu \mathrm{m}$ graded index (GI) multimode fiber and the output light from the multimode waveguide was end-fire coupled to a photodetector (Newport 818-UV) for the test of optical performance. The measured average losses of the single-reflection and the double-reflection mirror were 2.1 and $1.2 \mathrm{~dB}$, respectively. The double-reflection mirror results in lower optical loss than the single-reflection mirror, as expected [9]. It is important to lower the loss of the micromirrors because the mirror loss directly affects the insertion loss of arrayed waveguides.

Fig. 7 shows the insertion losses and the crosstalks of the 16-channel waveguide arrays. The insertion loss of the arrayed waveguides with the double-reflection mirrors is lower than that with the single-reflection mirrors due to the low loss of the double-reflection mirrors. The average insertion losses of the arrayed waveguides with the single- and double-reflection mirrors are 6.1 and $4.4 \mathrm{~dB}$, respectively. The measured insertion losses are sufficient to satisfy the optical budget in practical chip-level systems [15].

The coupling loss between the fiber and the waveguides can be estimated from the insertion loss, which is the sum of the propagation loss, mirror loss, and coupling loss. It is about $0.4 \mathrm{~dB}$ with the separately measured propagation loss of $0.3 \mathrm{~dB} / \mathrm{cm}$ [10]. The optical uniformities of the waveguide arrays with the single and double reflection mirrors are \pm 0.4 and $\pm 0.8 \mathrm{~dB}$, respectively. If the variation of the coupling loss due to the positional errors of the stacked waveguides is included, the optical loss uniformities will be increased up to \pm 1.9 and $\pm 2.2 \mathrm{~dB}$, respectively. Although the optical uniformities of the stacked waveguide arrays are not as good as other methods such as microlenses $( \pm 1.25 \mathrm{~dB})$ [13] and fiber image guiding $( \pm 1 \mathrm{~dB})$ [14], the stacked waveguide arrays have large potential because the optical uniformities can be reduced by the development of stacking process to reduce the positional errors.

Crosstalk between the waveguides must be considered in the waveguide array structures because the radiated light from a waveguide can be guided by nearby waveguides, which have a spacing of $50 \mu \mathrm{m}$. The radiation must be significant near the reflection mirrors, and higher reflection efficiency must result in lower crosstalk. The crosstalk in the arrays is displayed in Fig. 7. Light was coupled to channel 8 in the figure. The maximum crosstalks between the nearest waveguides with the single- and with the double-reflection mirrors were less than -26 and $-28 \mathrm{~dB}$, respectively, as shown in Fig. 7(b). The fluctuation of the crosstalk for different waveguides is less 


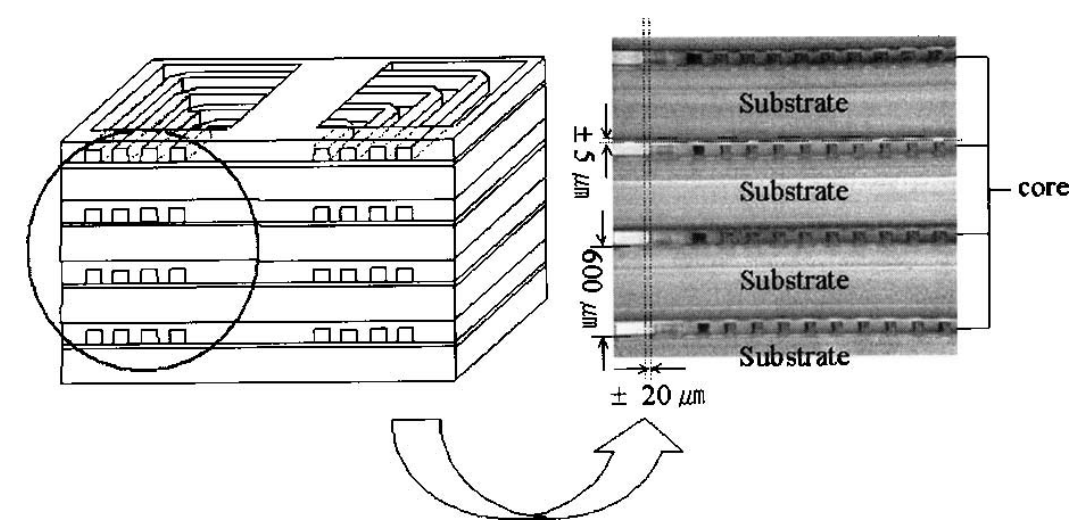

Fig. 6. Four-layer polymer waveguide array for the optical interconnects of a 2-D VCSEL array. The vertical spacing can be easily adjusted by controlling the thickness of the substrate or the cladding layer.

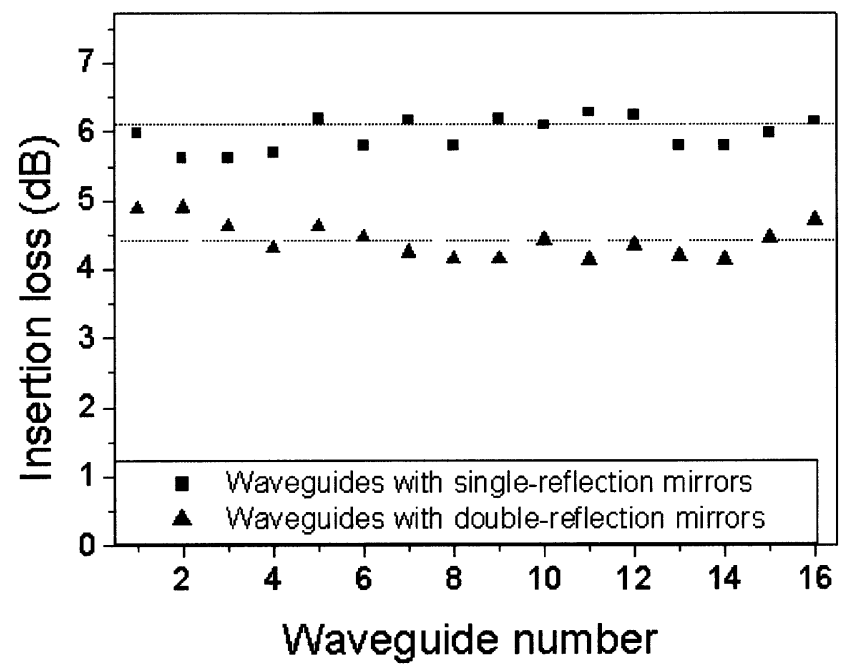

(a)

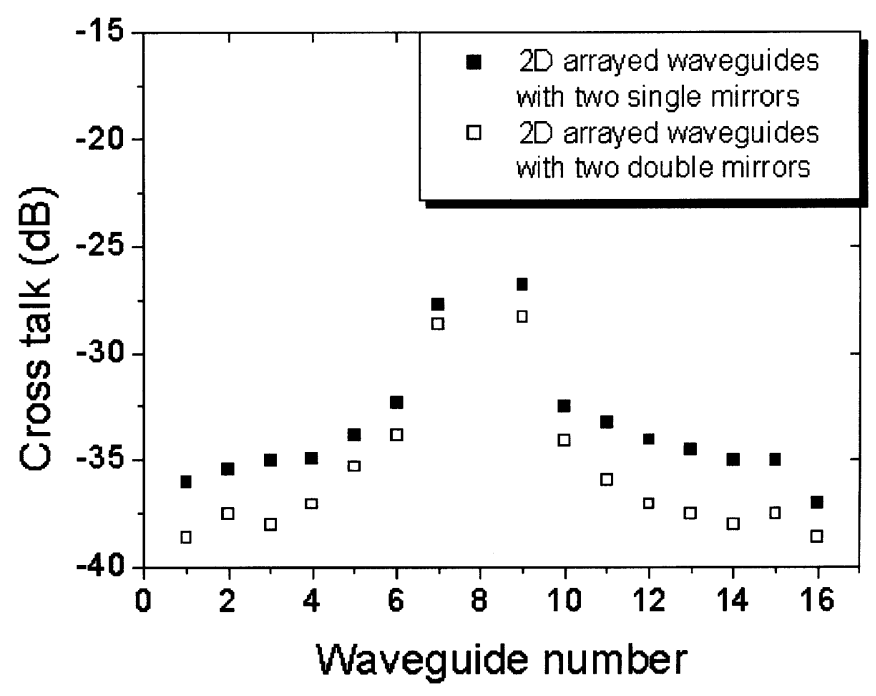

(b)

Fig. 7. (a) Insertion loss and (b) the crosstalk of the arrayed waveguides with the single- and double-reflected mirrors, respectively.

than $\pm 1.4 \mathrm{~dB}$. Lower crosstalk in the waveguides with the double-reflection mirrors is consistent with the lower mirror loss than the single-reflection mirrors.
The crosstalk between the stacked waveguides is less than $-35 \mathrm{~dB}$ between the closest waveguides. The low crosstalk is expected because the vertical spacing of the stacked waveguides is $600 \mu \mathrm{m}$, which is 12 times larger than the lateral spacing $(50 \mu \mathrm{m})$ between wavguides in the nonstacked waveguides. The crosstalk is low enough to use the 2-D waveguide arrays as 2-D optical interconnects. The pitches of VCSEL and PD arrays are $250 \mu \mathrm{m}$ or larger in general. In some cases, however, VCSELs and PDs with $125-\mu \mathrm{m}$ pitch were employed for high interconnection density [1]. Even in the case, the optical crosstalk in this stacked waveguide arrays is expected not to increase much because the closest spacing between the waveguides in the arrays is $50 \mu \mathrm{m}$, which is much smaller than $150-\mu \mathrm{m}$ pitch.

\section{CONCLUSION}

Novel 2-D arrayed polymeric multimode waveguides $\left(50 \times 50 \mu \mathrm{m}^{2}\right)$ with two reflection mirrors have been fabricated. Contact printing lithography was used for simple and low-cost process using a UV-curable epoxy-based polymer (SU-8) as the core layer. The material gives low optical loss at the wavelength of $830 \mathrm{~nm}$ and possesses thermal and chemical stability. The fabricated waveguides were diced of the same size and stacked one by one with lateral positional error less than $\pm 20 \mu \mathrm{m}$. The average optical losses from the single-reflection mirror and the double-reflection mirror were 1.2 and $2.1 \mathrm{~dB}$, respectively. The average insertion losses of 16-channel arrayed waveguides with single- and double-reflection mirrors were measured to be 6.1 and $4.4 \mathrm{~dB}$ for $6-\mathrm{cm}$-long waveguides at a wavelength of $830 \mathrm{~nm}$, respectively, which is good enough for practical optical interconnects. The fabricated 2-D arrayed polymeric multimode waveguides are applicable for 2-D optical interconnects between VCSELs and detectors to increase the number of interconnection channels.

\section{REFERENCES}

[1] D. V. Plant, M. B. Venditti, E. Laprise, J. Faucher, K. Razavi, M. Chateauneuf, A. G. Kirk, and J. S. Ahearm, "256-channel bi-directional optical interconnect using VSCELs and photodiodes on CMOS," $J$. Lightwave Technol., vol. 19, pp. 1093-1103, Aug. 2001.

[2] G. Kim, X. Han, and R. T. Chen, "An 8-Gb/s optical backplane bus based on microchannel interconnects: Design, fabrication, and performance measurements," J. Lightwave Technol., vol. 18, pp. 1477-1486, Nov. 2000. 
[3] A. Neyer, B. Wittmann, and M. Johnck, "Plastic-optical-fiber-based parallel optical interconnects," IEEE J. Select. Topics Quantum Electron., vol. 5, pp. 193-200, Mar.-Apr. 1999.

[4] Y. Li, J. Ai, and J. Popelek, "Board-level 2-D data-capable optical interconnection circuits using polymer fiber-image guides," Proc. IEEE, vol. 88, pp. 794-805, 2000.

[5] S. Lehmacher and A.Andreas Neyer, "Integration of polymer optical waveguides into printed circuit boards," Electron. Lett., vol. 36, pp. 1052-1053, 2000.

[6] Y. Ishii, S. Koike, Y. Arai, and Y. Ando, "SMT-compatible optical-I/O chip packaging for chip-level optical interconnects," in Proc. IEEE 51st ECTC, 2001, pp. 870-875.

[7] J. J. Maki, B. Booth, and R. T. Chen, "Three dimensional integrated polymer thin-film waveguides for high-density true-time-delay lines for phased-array-antenna applications," in Proc. SPIE, vol. 4292, 2001, pp. $62-72$.

[8] Y. Z. Tang, W. H. Wang, T. Li, and Y. L. Wang, "Integrated waveguide turning mirror in silicon-on-insulator," IEEE Photon. Technol. Lett., vol. 14, pp. 68-70, Jan. 2002.

[9] T. Namba, A. Uehara, T. Doi, T. Nagata, and Y. Kuroda, "High-efficiency micromirrors and branched optical waveguides on Si chips," Jpn. J. Appl. Phys., vol. 35, pp. 941-945, 1996.

[10] J.-S. Kim, J.-W. Kang, and J.-J. Kim, "Simple and low cost fabrication of thermally stable polymeric multimode waveguides using a UV-curable epoxy," Jpn. J. Appl. Phys., vol. 42, pp. 1277-1279, 2003.

[11] T. Cooesemans, A. V. Hove, K. Naessens, L. Vanwassenhove, P. V. Daele, and R. Baets, "Fabrication of a 2D connector for coupling a $4 \times 8$ arrays of small diameter plastic optical fiber $(117 / 125 \mu \mathrm{m})$ to MCLED or VCSEL arrays," in Proc. 50th Electronic Components Technology Conf. (ECTC), 2000, pp. 1236-1241.

[12] C. R.Clifford R. Pollock, "Coupling between optical sources and waveguides," in Fundamentals of Optoelectronics. Homewood, IL: Irwin, 1995, pp. 299-308.

[13] M. Chateruneuf, A. G. Kirk, D. V. Plant, T. Yamamoto, and J. D. Ahearn, "512-channel vertical-cavity surface-emitting laser based free-space optical link," Appl. Opt., vol. 41, pp. 5552-5561, 2002.

[14] T. Maj, A. G. Kirk, D. V. Plant, J. F. Ahadian, C. G. Fonstad, K. L. Lear, K. Tatah, M. S. Robinson, and J. A. Trezza, "Interconnection of a two-dimensional array of vertical-cavity surface-emitting lasers to a receiver array by means of a fiber image guide," Appl. Opt., vol. 39, pp. 683-689, 2000.
[15] D. H. Hartman, "Optical interconnects at the multi-chip module level: A view from the system level," in Proc. 43rd Electronic Components Technology Conf. (ECTC), 1993, pp. 701-704.

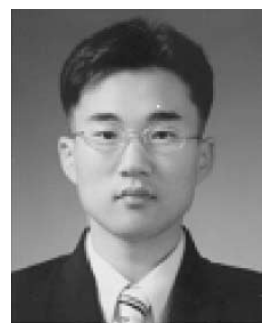

Joon-Sung Kim received the B.S. degree in inorganic materials engineering from Chonnam National University, Korea, in 1998 and the M.S. degree in materials science and engineering from Kwangju Institute of Science and Technology, Korea, in 2000, where he is currently pursuing the Ph.D. degree in materials science and engineering.

His research areas include polymer optical interconnection and optical waveguide devices from polymeric materials.

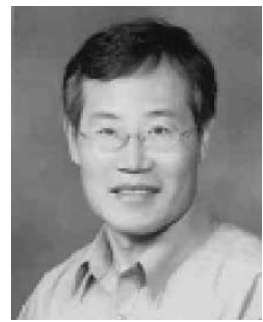

Jang-Joo Kim received the B.S. and M.S. degrees from the Department of Chemical Engineering, Seoul National University, Seoul, Korea, in 1977 and 1980, respectively, and the Ph.D. degree from the Department of Materials Science and Engineering, Stanford University, Stanford, CA, in 1987.

He was a Postdoctoral Fellow at SRI International during 1986-1987. He then returned to Korea to join ETRI as a Senior Member and a Principal Member of Technical Staff from 1987 to 1996 . He was a Professor at Kwangju Institute of Science and Technology, Korea, from 1997 to 2003. He is currently a Professor in the School of Materials Science and Engineering, Seoul National University. His research areas include polymer waveguide devices, plastic optical fiber, and organic electronics. He has published more than 90 papers and has received more than 30 patents. He has given more than 80 presentations to international conferences, including 30 invited talks. 